\title{
An Axiomatization of the Ratio/Difference Representation
}

\author{
JoHN M. MiYamoto \\ University of Michigan
}

\begin{abstract}
If $\geqslant_{r}$ and $\geqslant_{d}$ are two quaternary relations on an arbitrary set $A$, a ratio/difference representation for $\geqslant_{r}$ and $\geqslant_{d}$ is defined to be a function $f$ that represents $\geqslant_{r}$ as an ordering of numerical ratios and $\geqslant_{d}$ as an ordering of numerical differences. Krantz, Luce, Suppes and Tversky (1971, Foundations of Measurement. New York, Academic Press) proposed an axiomatization of the ratio/difference representation, but their axiomatization contains an error. After describing a counterexample to their axiomatization, Theorem 1 of the present article shows that it actually implies a weaker result: if $\geqslant_{r}$ and $\geqslant_{d}$ are two quaternary relations satisfying the axiomatization proposed by Krantz et al. (1971), and if $\geqslant_{r}$ and $\geqslant_{d}$ are the relations that are inverse to $\geqslant_{r}$ and $\geqslant_{d}$, respectively, then either there exists a ratio/difference representation for $\geqslant_{r}$ and $\geqslant_{d}$, or there exists a ratio/difference representation for $\geqslant_{r^{+}}$and $\geqslant_{d^{+}}$, but not both. Theorem 2 identifies a new condition which, when added to the axioms of Krantz et al. (1971), yields the existence of a ratio/difference representation for relations $\geqslant_{r}$ and $\geqslant_{d}$.
\end{abstract}

Garner (1954) suggested that one could determine a ratio scale for loudness if subjects are able to judge what stimulus is a given fraction as loud as another stimulus and if, in addition, they could partition a loudness interval into a given number of subintervals of equal subjective size. His proposal assumes that there are distinct mental operations that can be carried out on the subjective representation of loudness, the one operation being isomorphic to the calculation of numerical ratios and the second operation being isomorphic to the calculation of numerical differences. Torgerson (1961) doubted that there exist two distinct operations of loudness judgment. He proposed that even if subjects are instructed, on the one hand, to judge the magnitude of subjective ratios and, on the other hand, to judge the magnitude of subjective differences, the mental operations underlying their responses would be the same. Michael Birnbaum and his colleagues have carried out an extensive program of experimentation devoted to testing these opposing hypotheses. On the whole, their evidence supports the theory that only one mental operation underlies judgments of ratios and differences, although this conclusion has not been universally accepted. ${ }^{1}$ Birnbaum (1978, 1982), Hagerty and Birnbaum (1978) and

I would like to thank David H. Krantz for many valuable discussions of these and related ideas. Requests for reprints should be sent to John M. Miyamoto, Department of Psychology, University of Michigan, Ann Arbor, Mi. 48104-1346.

'Birnbaum $(1978,1980,1982)$ reviews cvidence and arguments favoring the theory that only one 
Veit (1978) have attempted to show that the sole underlying operation of magnitude judgment is a subtractive operation. Their arguments lie beyond the scope of the present discussion.

The purpose of this article is not to discuss these psychophysical issues, but rather to present an axiomatization of the theory that two mental operations of magnitude judgment exist. The following formulation of this theory is due to Krantz et al. (1971), although some of the terminology is my own. If there are distinct mental operations of magnitude judgment that are isomorphic, respectively, to the calculation of ratios and of differences, then subjects ought to order pairs of stimuli in two different ways depending on whether they are asked to judge the magnitude of subjective ratios or the magnitude of subjective differences. Furthermore, these two orderings of stimulus pairs ought to possess a common numerical representation as orderings, respectively, of ratios and of differences. This common representation will be called a ratio/difference representation and is described formally in Definition 1 . As a notational convention, if $y$ and $z$ denote elements of a set $A$, then $y z$ denotes the ordered pair $(y, z)$.

DEFINITION 1 . Let $\geqslant_{r}$ and $\geqslant_{d}$ be quaternary relations on an arbitrary set $A$. Let $\mathrm{Re}^{+}$denote the positive real numbers and let $f: A \rightarrow \operatorname{Re}^{+}$be any function. Then, $f$ is $a$ ratio/difference representation for $\geqslant_{r}$ and $\geqslant_{d}$ iff for all $w, x, y, z \in A$

(i) $\quad w x \geqslant_{r} y z$ iff $f(w) / f(x) \geqslant f(y) / f(z)$

(ii) $w x \geqslant_{d} y z$ iff $f(w)-f(x) \geqslant f(y)-f(z)$.

Thus, a ratio/difference representation is a function $f$ that simultaneously represents an ordering $\geqslant_{r}$ as an ordering of numerical ratios and an ordering $\geqslant_{d}$ as an ordering of numerical differences.

Before discussing the ratio/difference representation, it will be helpful to set down several conventions. First, $>_{r}$ and $>_{d}$ denote the strict inequalities and $\sim_{r}$ and $\sim_{d}$ denote the equivalence relations corresponding to $\geqslant_{r}$ and $\geqslant_{d}$, respectively. Furthermore, there is a trivial case that it is convenient to exclude, namely, if $w x \sim_{r} y z$ and $w x \sim_{d} y z$ for all $w, x, y, z \in A$, then any constant, positive real function is a ratio/difference representation for $\geqslant_{r}$ and $\geqslant_{d}$. Since this case is uninteresting, it will be assumed throughout this essay that $\geqslant_{r}$ and $\geqslant_{d}$ are nondegenerate in the sense that $s t>_{r} u v$ and $w x>_{d} y z$ for some $s, t, u, v, w, x, y, z \in A$.

Krantz et al. (1971) propose an axiomatization of the ratio/difference represen-

mental operation underlies judgments of subjective ratios and subjective differences. The theory originates with Torgerson (1961). Studies supporting this theory include Birnbaum and Elmasian (1978), Birnbaum and Mellers (1978), Birnbaum and Veit (1974), Hagerty and Birnbaum (1978), Mellers, Davis, and Birnbaum (1984), Schneider, Parker, Farrell, and Kanow (1976) and Veit (1978, 1980). The theory that there are distinct judgments of subjective ratio and difference applying to a single mental representation has been defended by Rule and Curtis (1980) and Rule, Curtis, and Mullin (1981). Parker, Schneider, and Kanow (1975) provide evidence that distinct judgments of subjective ratio and subjective difference can be performed on the continuum of perceived line lengths. Marks (1974) and Stevens $(1957,1971)$ develop views that do not fall into either camp. 
tation and construct a purported proof that the axiomatization is sufficient to establish the existence of the representation. Next, I will state their axiomatization, discuss its interpretation, and then describe a counterexample to the sufficiency of the axiomatization. Later, an analysis will be given that locates the error in the attempted proof of the representation theorem appearing in Krantz et al. (1971). The following definition makes use of the concept of an algebraic difference structure which is defined in Krantz et al. (1971, Definition 4.3).

DEFINITION 2. Let $\geqslant_{r}$ and $\geqslant_{d}$ be quaternary relations on an arbitrary set $A$. Then $\geqslant_{r}$ and $\geqslant_{d}$ satisfy generalized ratio/difference compatibility iff $\left(A \times A, \geqslant_{r}\right)$ and $\left(A \times A, \geqslant_{d}\right)$ are both algebraic difference structures and for all $x, y, z, x^{\prime}, y^{\prime}, z^{\prime} \in A$,

(i) $x y \geqslant_{r} x x$ iff $x y \geqslant_{d} x x$,

(ii) if $x x^{\prime} \sim_{r} y y^{\prime} \sim_{r} z z^{\prime}$, then $x y \geqslant_{d} y z$ iff $x^{\prime} y^{\prime} \geqslant_{d} y^{\prime} z^{\prime}$,

(iii) $\geqslant_{r}$ and $\geqslant_{d}$ are not identical orderings.

Generalized ratio/difference compatibility is proposed by Krantz et al. (1971) as an axiomatizaton of the ratio/difference representation, although they do not use this terminology (cf. the hypotheses of their Theorem 4.3, p. 154).

The assumption that $\left(A \times A, \geqslant_{r}\right)$ and $\left(A \times A, \geqslant_{d}\right)$ are both algebraic difference structures is quite plausible, since the existence of a ratio/difference representation implies that both structures have difference representations (the logarithm of the ratio representation is a difference representation). Intuitively, condition (i) asserts that $\geqslant_{r}$ and $\geqslant_{d}$ determine the same set of positive intervals. Condition (ii) is rather complicated in appearance, but it implies the more easily interpreted condition,

(ii $\left.i^{\prime}\right)$ if $x x^{t} \sim_{r} y y^{\prime} \sim_{r} z z^{\prime}$, then $x y \sim_{d} y z$ iff $x^{\prime} y^{\prime} \sim_{d} y^{\prime} z^{\prime}$.

Note that if $u v \sim_{d} v w$, then $v$ may be regarded as the $\geqslant_{d}$ midpoint between $u$ and $w$, i.e., the midpoint in the $\geqslant_{d}$ ordering. Thus, condition (ii') asserts that if $x x^{\prime}, y y^{\prime}$. and $z z^{\prime}$ are all in the same $\geqslant_{r}$ equivalence class, then $y$ is the $\geqslant_{d}$ midpoint between $x$ and $z$ iff $y^{\prime}$ is the $\geqslant_{d}$ midpoint between $x^{\prime}$ and $z^{\prime}$. Condition (ii) is just an ordinal version of (ii'). Krantz et al. (1971) show that conditions (i) and (ii) of Definition 2 are necessary for the ratio/difference representation. To see that condition (iii) must hold, suppose that $\geqslant_{r}$ and $\geqslant_{d}$ were identical. If $f$ were a ratio/difference representation for $\geqslant_{r}$ and $\geqslant_{d}$, then $\log f$ would be a difference representation for $\geqslant_{r}$, so $\log f$ and $f$ would be difference representations for $\geqslant_{d}$. By the uniqueness of the algebraic difference representation for $\left(A \times A, \geqslant_{d}\right)$, there must be constants $\alpha, \beta$ such that $\log f=\alpha f+\beta$. It can be shown that $f(A)$ is dense in an interval of real numbers ${ }^{2}$ and consequently there exist $x, y, z \in A$ such that $f(x) \neq f(y) \neq f(z) \neq f(x)$. Since a linear relation between $\log f$ and $f$ cannot be obtained if the range of $f$ has more than two values, $\geqslant_{r}$ and $\geqslant_{d}$ cannot be identical.

\footnotetext{
${ }^{2}$ The set $f(A)$ is dense in an interval of real numbers iff for every $x$ and $z$ in that interval, $x<z$ implies that there exists $y \in f(A)$ such that $x<y<z$. The proof that $f(A)$ is dense in an interval of reals is given in Krantz et al. (1971. p. 159).
} 
An important feature of generalized ratio-difference compatibility is that if it holds for a pair of relations $\geqslant_{r}$ and $\geqslant_{d}$, then it must also hold for the corresponding inverse relations. In other words, define relations $\geqslant_{r^{*}}$ and $\geqslant_{d^{*}}$ by the conditions

$$
\begin{array}{ll}
w x \geqslant_{r^{*}} y z & \text { iff } y z \geqslant_{r} w x, \\
w x \geqslant_{d^{*}} y z & \text { if } y z \geqslant_{d} w x,
\end{array}
$$

for every $w, x, y, z \in A$. I claim that if $\geqslant_{r}$ and $\geqslant_{d}$ satisfy generalized ratio/difference compatibility, then $\geqslant_{r^{*}}$ and $\geqslant_{d^{*}}$ must also satisfy generalized ratio/difference compatibility. Assuming that $\geqslant_{r}$ and $\geqslant_{d}$ satisfy generalized ratio/difference compatibility, it is easy to check that $\left(A \times A, \geqslant_{r^{*}}\right)$ and $\left(A \times A, \geqslant_{d^{*}}\right)$ are algebraic difference structures and that $\geqslant_{r^{*}}$ and $\geqslant_{d^{*}}$ satisfy conditions (i) and (iii) of Definition 2. As for condition (ii), the pairs $x x^{\prime}, y y^{\prime}$ and $z z^{\prime}$ are in the same $\geqslant_{\text {r }}$ equivalence class iff they are in the same $\geqslant_{r^{*}}$ equivalence class. So the antecident of (ii) holds with respect to $\geqslant_{r}$ iff it holds with respect to $\geqslant_{r^{*}}$. The consequent of (ii) asserts that $x y \geqslant_{d} y z$ iff $x^{\prime} y^{\prime} \geqslant_{d} y^{\prime} z^{\prime}$, but this is equivalent to asserting that $x y \geqslant_{d^{*}} y z$ iff $x^{\prime} y^{\prime} \geqslant_{d^{*}} y^{\prime} z^{\prime}$. So if the consequent of (ii) holds with respect to $\geqslant_{d}$, it also holds with respect to $\geqslant_{d^{*}}$.

Thus, the definition of generalized ratio/difference compatibility possesses a kind of symmetry with respect to the relations $\geqslant_{r}$ and $\geqslant_{d}$, and the inverse relations $\geqslant_{r *}$ and $\geqslant_{d^{*}}$. This symmetry is the crucial defect in the conjecture that generalized ratio/difference compatibility implies the existence of a ratio/difference representation. Consider the following counterexample to this conjecture. Let $A=\mathrm{Re}^{+}$. Define relations $R$ and $D$ by the conditions: for any $w, x, y, z \in \operatorname{Re}^{\prime}$,

$$
\begin{array}{ll}
w x R y z & \text { if } \quad w / x \geqslant y / z, \\
w x D y z & \text { if } \quad w-x \geqslant y-z,
\end{array}
$$

where / and - denote division and subtraction of real numbers. Obviously, $R$ and $D$ satisfy generalized ratio/difference compatibility and the identity function $I: \mathrm{Re}^{+} \rightarrow \mathrm{Re}^{+}$is a ratio/difference representation for $R$ and $D$. Let $R^{*}$ and $D^{*}$ denote the relations inverse to $R$ and $D$. By a previous argument, $R^{*}$ and $D^{*}$ must satisfy generalized ratio/difference compatibility.

Suppose that generalized ratio/difference compatibility were sufficient to imply the existence of a ratio/difference representation, and let $f$ be the ratio/difference representation for $R^{*}$ and $D^{*}$. Since $D^{*}$ is inverse to $D,-f$ must be a difference reprcsentation for $D$. Hence $-f=\eta I+\lambda$ for $\eta \in \operatorname{Re}^{+}$and $\lambda \in$ Re. Similarly, since $R^{*}$ is inverse to $R, 1 / f$ must be a ratio representation for $R$. Hence, $1 / f=\alpha I^{B}$ for some $\alpha, \beta \in \mathrm{Re}^{+}$. But then, for every $x \in \mathrm{Re}^{+}$,

$$
-\eta x-\lambda=-\eta I(x)-\lambda=f(x)=1 / \alpha I(x)^{\beta}=1 / \alpha x^{3} .
$$

Hence, $-\eta \alpha x^{\beta+1}-\lambda \alpha x^{\beta}=1$ for all $x \in \operatorname{Re}^{+}$. Obviously, this identity cannot be satisfied within the constraints on $\eta, \lambda, a$, and $\beta$. Therefore, there cannot exist a ratio/difference representation $f$ for $R^{*}$ and $D^{*}$, so generalized ratio/difference 
compatibility is not sufficient to imply the existence of a ratio/difference representation.

It should now be plausible that a more general statement of the counterexample also holds. Namely, if $\geqslant_{r}$ and $\geqslant_{d}$ are any relations satisfying generalized ratio/difference compatibility, then their respective inverses $\geqslant_{r^{*}}$ and $\geqslant_{d^{*}}$ also satisfy generalized ratio/difference compatibility. If generalized ratio/difference compatibility were sufficient to imply the existence of a ratio/difference representation, then both pairs of relations, $\geqslant_{r}$ and $\geqslant_{d}$, and $\geqslant_{r^{*}}$ and $\geqslant_{d^{*}}$, would possess ratio/difference representations. The essence of the counterexample is to show that this cannot be. At most one pair of relations, $\geqslant_{r}$ and $\geqslant_{d}$, or the inverses $\geqslant_{r^{*}}$ and $\geqslant_{d^{*}}$, could have a ratio/difference representation. Theorem 1 asserts that exactly one pair of relations, $\geqslant_{r}$ and $\geqslant_{d}$, or $\geqslant_{r^{*}}$ and $\geqslant_{d^{*}}$, has a ratio/difference representation if either pair satisfies generalized ratio/difference compatibility.

THEOREM 1. Let $\geqslant_{r}$ and $\geqslant_{d}$ be quaternary relation on an arbitrary set $A$, and let $\geqslant_{r^{*}}$ and $\geqslant_{d^{*}}$ be the respective inverse relations. If $\geqslant_{r}$ and $\geqslant_{d}$ satisfy generalized ratio/difference compatibility, then either there exists a ratio/difference representation f for $\geqslant_{r}$ and $\geqslant_{d}$, or there exists a ratio/difference representation $g$ for $\geqslant_{r^{*}}$ and $\geqslant_{d^{*}}$, but not both. Moreover, if $f$ or $g$ exists, then it is a ratio scale.

The proof of Theorem 1 is based on Lemma 1 stating the solution to a functional equation.

LEMMA 1. Let $I_{1}$ be a nonempty interval of real numbers and let $I_{2}$ be a nonempty interval of positive real numbers. If $H: I_{2} \rightarrow I_{1}$ is a continuous, strictly increasing function satisfying

$$
H^{-1}\left[\frac{H(t x)+H(t y)}{2}\right]=t \cdot H^{-1}\left[\frac{H(x)+H(y)}{2}\right]
$$

for all $x, y \in I_{2}$ and for any $t \in \mathrm{Re}^{+}$such that $t x, t y \in I_{2}$, then either (i) or (ii) holds:

(i) $H(x)=\alpha+\eta \log x$ for all $x \in I_{2}$, where $\alpha \in \mathrm{Re}, \eta \in \mathrm{Re}^{+}$,

(ii) $H(x)=\delta \alpha x^{\delta \theta}-\beta$ for all $x \in I_{2}$, where $\alpha, \theta \in \operatorname{Re}^{+}, \beta \in \operatorname{Re}$ and $\delta=+1$ or -1 .

Aczel (1966, Sect. 3.1.3) proves Lemma 1 for the case where $I_{2}=\mathrm{Re}^{+}$. Krantz et al. (1971, Sect. 4.5.3) develop the present generalization of the lemma, except that their proof appears to rule out the possibility in solution (ii) that $\delta=-1$. That this is an oversight can be established by checking that $H(x)=-x^{-1}$ is a function satisfying (1). The proof of Lemma 1 will be given in the Appendix because it is rather lengthy. Readers who wish to skip the proof may nevertheless want to note that the error in the proof of Krantz et al. (1971) occurs on p. 163 where it is inferred that a function called $\gamma_{1}$ is strictly increasing. As shown in the Appendix, $\gamma_{1}$ can either be strictly decreasing or strictly increasing. 
The proof of Theorem 1 presented here is very close to the proof of Theorem 4.3 in Krantz et al. (1971, pp. 158-163), except for the modifications resulting from the reformulation of Lemma 1 . To abbreviate the many references to Krantz et al. (1971), this work will be referred to as Fnd in the remainder of this article.

Proof of Theorem 1. Let $\phi_{1}$ be a difference representation for $\left(A \times A, \geqslant_{d}\right)$ and let $\phi_{2}$ be a ratio representation for $\left(A \times A, \geqslant_{r}\right)$. Notice for future reference that $-\phi_{1}$ must be a difference representation for $\left(A \times A, \geqslant_{d^{*}}\right)$ and $1 / \phi_{2}$ must be a ratio representation for $\left(A \times A, \geqslant_{r^{*}}\right)$. Let $R_{i}=\phi_{i}(A), i=1,2$. Define $h: R_{2} \rightarrow R_{1}$ by $h\left[\phi_{2}(x)\right]=\phi_{1}(x)$ for any $x \in A$. The function $h$ is well defined because condition (i) of Definition 2 implies that $\phi_{2}(x)=\phi_{2}(y)$ iff $x y \sim_{r} x x$ iff $x y \sim_{d} x x$ iff $\phi_{1}(x)=\phi_{1}(y)$.

It can be prove tht there exist intervals $I_{1} \subseteq \operatorname{Re}$ and $I_{2} \subseteq \mathrm{Re}^{+}$and a strictly increasing, continuous function $H: I_{2} \rightarrow I_{1}$ such that $R_{i}$ is a dense subset of $I_{i}$ $(i=1,2), H\left[\phi_{2}(x)\right]=h\left[\phi_{2}(x)\right]$ for all $x \in A$, and

$$
H^{-1}\left[\frac{H(t u)+H(t v)}{2}\right]=t \cdot H^{-1}\left[\frac{H(u)+H(v)}{2}\right]
$$

for all $u, v \in I_{2}$ and $t \in \mathrm{Re}^{+}$for which $t u, t v \in I_{2}$ (see Fnd, pp. 159-160, for proofs of these properties). Lemma 1 states the possible solutions of this functional equation.

Solution (i) of Lemma 1 implies that $\phi_{1}(x)=H\left[\phi_{2}(x)\right]=\alpha+\eta \cdot \log \phi_{2}(x)$ for all $x \in A$. Hence $\phi_{1}$ is a difference representation for $\left(A \times A, \geqslant_{r}\right)$, because $\phi_{2}$ is a ratio representation for that structure. Since $\phi_{1}$ is also a difference representation for $\left(A \times A, \geqslant_{d}\right)$, the orderings $\geqslant_{r}$ and $\geqslant_{d}$ must be identical, contrary to assumption. Therefore, solution (i) must be excluded.

Solution (ii) of Lemma 1 implies that $\phi_{1}(x)=H\left[\phi_{2}(x)\right]=\delta \alpha \phi_{2}(x)^{\delta \theta}-\beta$ for every $x \in A$. If $\delta=+1$, then $\phi_{1}(x)+\beta=\alpha \phi_{2}(x)^{\theta}$ for all $x \in A$. Therefore, $f=\phi_{1}+\beta=\alpha \phi_{2}^{\theta}$ is the desired ratio/difference representation for $\geqslant_{r}$ and $\geqslant_{d}$. If $f^{\prime}$ is any other ratio/ difference representation for $\geqslant_{r}$ and $\geqslant_{d}$, then $f^{\prime}=\gamma f^{\lambda}$ for $\gamma, \lambda \in \operatorname{Re}^{+}$because $f^{\prime}$ and $f$ are both ratio representations for $\geqslant_{r}$, and $f^{\prime}=\pi f+\omega$ for $\pi \in \operatorname{Re}^{+}$and $\omega \in \operatorname{Re}$ because $f^{\prime}$ and $f$ are both difference representations for $\geqslant_{d}$. Hence $\gamma f^{\lambda}-\pi f-\omega \equiv 0$ (where $\equiv$ signifies "is constantly equal to"), so $\lambda=1, \gamma=\pi$, and $\omega=0$. Thus, $f^{\prime}=\pi f$ so $f$ is a ratio scale. If $\delta=-1$, then $-\phi_{1}(x)-\beta=\alpha\left[1 / \phi_{2}(x)\right]^{\theta}$ for all $x \in A$. Since $-\phi_{1}$ is a difference representation for $\geqslant_{d^{*}}$ and $1 / \phi_{2}$ is a ratio representation for $\geqslant_{r^{*}}$, we have $g=-\phi_{1}-\beta=\alpha\left[1 / \phi_{2}\right]^{\theta}$ as the ratio/difference representation for $\geqslant_{r^{*}}$ and $\geqslant_{d^{*}}$. The proof that $g$ is a ratio scale is the same as the proof for $f$.

There cannot exist both a ratio/difference representation $f$ for $\geqslant_{r}$ and $\geqslant_{d}$ and a ratio/difference representation $g$ for $\geqslant_{r^{*}}$ and $\geqslant_{d^{*}}$, for suppose they both existed. Then $1 / g$ would be a ratio representation for $\geqslant_{r}$ and $-g$ would be a difference representation for $\geqslant_{d}$. By the uniqueness of these representations, $f=-\alpha g-\beta=\eta(1 / g)^{\lambda}$ for $\alpha, \eta, \lambda \in \operatorname{Re}^{+}$and $\beta \in \mathrm{Re}$. Hence

$$
-\alpha g^{\lambda+1}-\beta g^{\lambda}-\eta \equiv 0 .
$$


Since $g(A)$ is dense in a nonempty interval of real numbers (see Fnd, p. 159 for the proof), there are no $\alpha, \lambda \in \operatorname{Re}^{+}$for which (2) is satisfied. So $f$ and $g$ cannot both exist.

Q.E.D.

It is clear from Theorem 1 that the ratio/difference representation can be axiomatized by supplementing generalized ratio/difference compatibility with conditions that distinguish between the case where a representation exists for $\geqslant_{r}$ and $\geqslant_{d}$, and the case where a representation cxists for $\geqslant_{r^{*}}$ and $\geqslant_{d^{*}}$. Such conditions will now be described. Suppose that $\geqslant_{r}$ and $\geqslant_{d}$ satisfy generalized ratio/difference compatibility, that $\phi$ is a ratio representation for $\geqslant_{r}$ and that $\psi$ is a difference representation for $\geqslant_{d}$. Consider the possible relations between $\phi$ and $\psi$. There exists a ratio/difference representation $f$ for $\geqslant_{r}$ and $\geqslant_{d}$ iff $f$ is a ratio representation for $\geqslant_{r}$ and $f$ is a difference representation for $\geqslant_{d}$, i.e., iff $\alpha \phi^{\beta}=f=\lambda \psi+\tau$ for $\alpha, \beta, \lambda \in \mathrm{Re}^{+}$and $\tau \in \mathrm{Re}$. Therefore, the existence of a ratio/difference representation $f$ is equivalent to the existence of constants $\eta=\alpha / \lambda \in \mathrm{Re}^{+}$and $\mu=-\tau / \lambda \in \operatorname{Re}$ such that

$$
\psi=n \phi^{\beta}+\mu \text {. }
$$

By the same reasoning, there exists a ratio/difference representation $g$ for $\geqslant_{r^{*}}$ and $\geqslant_{d^{*}}$ iff $\alpha \phi^{-\beta}=g=-\lambda \psi+\tau$ for $\alpha, \beta, \lambda \in \operatorname{Re}^{+}$and $\tau \in \mathrm{Re}$, and this is equivalent to

$$
\psi=-\eta \phi^{-\beta}+\mu
$$

for $\eta=\alpha / \lambda \in \operatorname{Re}^{+}$and $\mu=\tau / \lambda \in \operatorname{Re}$. Furthermore, it can be shown that

$$
\psi=\eta[\log \phi]+\mu
$$

for $\eta \in \mathrm{Re}^{+}$and $\mu \in \operatorname{Re}$ is equivalent to the hypothesis that, with the exception of condition (iii), all assumptions of generalized ratio/difference compatibility are satisfied, i.e., $\geqslant_{r}$ and $\geqslant_{d}$ are identical orderings, $\left(A \times A, \geqslant_{r}\right)$ is an algebraic difference structure and conditions (i) and (ii) of Definition 2 hold.

Fagot (1963) points out that there is a simple way to distinguish between (3), (4), and (5). To describe the appropriate diagnostic property, it will be helpful to introduce some temporary existential assumptions. Later, these assumptions will be dropped once the basic ideas have been explained. Suppose that for every $w, z \in A$, there exist $\mathbf{a} \geqslant_{r}$ midpoint and $\mathbf{a} \geqslant_{d}$ midpoint in the sense that there exist $u, v \in A$ such that $w u \sim_{r} u z$ and $w v \sim_{d} v z$. If this is the case, one can define binary operations ${ }_{r}$ and $\left.\right|_{d}$ on $A$ by selecting for each $w, z \in A$, elements $\left.w\right|_{r} z$ and $\left.w\right|_{d} z$ satisfying

$$
\begin{aligned}
& w\left(\left.w\right|_{r} z\right) \sim_{r}\left(\left.w\right|_{r} z\right) z, \\
& w\left(\left.w\right|_{d} z\right) \sim_{d}\left(\left.w\right|_{d} z\right) z .
\end{aligned}
$$

By definition, $\left.w\right|_{r} z$ is a $\geqslant_{r}$ midpoint between $w$ and $z$, while $\left.w\right|_{d} z$ is a midpoint between $w$ and $z$. Given a ratio representation $\phi$ for $\geqslant_{r}$ and a difference representation $\psi$ for $\geqslant_{d}$, the relations (6) and (7) imply that $\phi(w) / \phi\left(\left.w\right|_{r} z\right)=\phi\left(\left.w\right|_{r} z\right) / \phi(z)$ and $\psi(w)-\psi\left(\left.w\right|_{d} z\right)=\psi\left(\left.w\right|_{d} z\right)-\psi(z)$. Thus, $\quad \phi\left(\left.w\right|_{r} z\right)=[\phi(w) \phi(z)]^{1 / 2} \quad$ and 
$\psi\left(\left.w\right|_{d} z\right)=[\psi(w)+\psi(z)] / 2$. In other words, $\phi\left(\left.w\right|_{r} z\right)$ is the geometric mean of $\phi(w)$ and $\phi(z)$, while $\psi\left(\left.w\right|_{d} z\right)$ is the arithmetic mean of $\psi(w)$ and $\psi(z)$. Fagot (1963) points out that (3)-(5) can be distinguished by the conditions:

(a) Equation (3) holds iff $\phi\left(\left.w\right|_{d} z\right)>\phi\left(\left.w\right|_{r} z\right)$ for all $w, z \in A$ such that $\phi(w) \neq \phi(z)$.

(b) Equation (4) holds iff $\phi\left(\left.w\right|_{d} z\right)<\phi\left(\left.w\right|_{r} z\right)$ for all $w, z \in A$ such that $\phi(w) \neq \phi(z)$.

(c) Equation (5) holds iff $\phi\left(\left.w\right|_{d} z\right)=\phi\left(\left.w\right|_{r} z\right)$ for all $w, z \in A$.

Thus (3)-(5) are distinguished by whether for every pair $w, z \in A$ of nonequivalent stimuli, $\phi$ applied to the $\geqslant_{d}$ midpoint of $w$ and $z$ is strictly greater than, strictly less than, or equal to the geometric mean of $\phi(w)$ and $\phi(z)$.

Since (3) implies that a ratio/difference representation exists for $\geqslant_{r}$ and $\geqslant_{d}$, the equivalence in (a) shows that the ratio/difference representation can be axiomatized by augmenting generalized ratio/difference compatibility with a qualitative axiom guaranteeing that $\phi\left(\left.w\right|_{d} z\right)>\phi\left(\left.w\right|_{r} z\right)$ for all $w, z \in A$ such that $\phi(w) \neq \phi(z)$. The following postulate formalizes this property:

(d) For all $w, z \in A$, if $w z \chi_{r} z z$, then $\left(\left.w\right|_{d} z\right)\left(\left.w\right|_{r} z\right)>_{r} z z$.

If (d) is added to Definition 2 , and if $\geqslant_{r}$ and $\geqslant_{d}$ midpoints exist for every pair of elements, then $\geqslant_{r}$ and $\geqslant_{d}$ possess a ratio/difference representation. Although this statement should be qualified in ways described below, one can say heuristically that the error in the axiomatization stated in Krantz et al. (1971) is that it fails to assert that $\geqslant_{d}$ midpoints of nonequivalent stimuli must strictly exceed corresponding $\geqslant_{r}$ midpoints.

Rather than to include (d) in an axiomatization of the ratio/difference representation, however, it is preferable to weaken (d) in ways that increase both the generality and the empirical testability of the axiomatization. Assuming that $\geqslant_{r}$ and $\geqslant_{d}$ satisfy generalized ratio/difference compatibility, either (3) or (4) must hold. But if there exists even one pair $w, z \in A$ such that $\phi(w) \neq \phi(z)$ and $\phi\left(\left.w\right|_{d} z\right)>\phi\left(\left.w\right|_{r} z\right)$, then (b) implies that (4) is false. But then, (3) must be true, so a ratio/difference representation exists for $\geqslant_{r}$ and $\geqslant_{d}$. This argument suggests the postulate:

(e) There exist $w, z \in A$ such that $\left(\left.w\right|_{d} z\right)\left(\left.w\right|_{r} z\right)>_{r} z z$ and $w z \chi_{r} z z$.

If (e) is added to the assumptions of Definition 2 , then $\geqslant_{r}$ and $\geqslant_{d}$ possess a ratio/difference representation. The advantage of (e) over (d) is that it only requires that $a \geqslant_{d}$ midpoint exceed $a \geqslant_{r}$ midpoint for one pair of nonequivalent stimuli, and hence, its empirical verification is simpler than that of (d).

The only shortcoming of (e) is that it requires that $a \geqslant_{r}$ midpoint and $a \geqslant_{d}$ midpoint exist for at least one pair $w, z \in A$ such that $w z \chi_{d} z z$. Since this existential claim might be difficult to establish in certain applications of the theory, it would be preferable to weaken it. To see the appropriate weakening, appeal must be made to the following technical point. It is proven in Fnd (p. 159) that if $\left(A \times A, \geqslant_{r}\right)$ and 
$\left(A \times A, \geqslant_{d}\right)$ are algebraic difference structures, and if $\geqslant_{r}$ and $\geqslant_{d}$ possess a ratio/difference representation, then there exist intervals $I_{r}$ and $I_{d}$ of real numbers such that $\phi(A)$ is dense in $I_{r}$ and $\psi(A)$ is dense in $I_{d} .{ }^{3}$ Therefore, even if midpoints $\left.w\right|_{r} z$ and $\left.w\right|_{d} z$ do not exist, there must exist elements that are arbitrarily close to the midpoint locations in the sense that for any $\varepsilon>0$ there exist $p, q \in A$ such that $\varepsilon>\mid \log \phi(p)-$ $\log [\phi(w) \phi(z)]^{1 / 2} \mid$ and $\varepsilon>|\psi(q)-[\psi(w)+\psi(z)] / 2|$. It should be plausible that (e) can be reformulated using elements that are close to if not precisely at the $\geqslant_{r}$ and $\geqslant_{d}$ midpoints of $w$ and $z$. The desired condition is stated in:

(f) There exist $w, x, y, z \in A$ such that $w x \geqslant_{d} x z, w y \leqslant r y z, w z \downarrow_{r} z z$ and $x y>_{r} x x$.

Intuitively, the elements $x$ and $y$ of (f) may be thought of as having been chosen to satisfy $\varepsilon>[\psi(w)+\psi(z)] / 2-\psi(x)>0$ and $\varepsilon>\log \phi(y)-\log [\phi(w) \phi(z)]^{1 / 2}>0$ for some very small $\varepsilon$. In other words, $x$ is close to and below the $\geqslant_{d}$ midpoint of $w$ and $z$, and $y$ is close to and above the $\geqslant$ midpoint of $w$ and $z$.

Theorem 2 asserts that if $\left(A \times A, \geqslant_{r}\right)$ and $\left(A \times A, \geqslant_{d}\right)$ are algebraic difference structures such that $\geqslant_{r}$ and $\geqslant_{d}$ possess a ratio/difference representation, then (f) must be satisfied. Indeed, for any choice of $w, z \in A$ such that $w z f_{r} z z$, there exist $x, y \in A$ satisfying (f). Conversely, if $\geqslant_{r}$ and $\geqslant_{d}$ satisfy (f) and generalized ratio/difference compatibility, then a ratio/difference representation exists for $\geqslant_{r}$ and $\geqslant_{d}$. Before stating the representation theorem, it will be useful to state and prove a lemma showing that (f) implies condition (iii) of Definition 2. This lemma permits the substitution of (f) for condition (iii) of Definition 2 in the axiomatization of the ratio/difference representation.

Lemma 2. Let $\left(A \times A, \geqslant_{d}\right)$ and $\left(A \times A, \geqslant_{r}\right)$ be algebraic difference structures satisfying conditions (i) and (ii) of Definition 2. If there exist $w, x, y, z \in A$ such that $w x \geqslant_{d} x z, w y \leqslant_{r} y z, w z \chi_{r} z z$ and $x y>_{r} x x$, then $\geqslant_{d}$ and $\geqslant_{r}$ are not identical.

Proof. Since $\left(A \times A, \geqslant_{d}\right)$, is an algebraic difference structure, there exists a difference representation $f$ for $\geqslant_{d}$. Using the difference representation, it readily follows that if $a b>_{d} a^{\prime} b^{\prime}$, then $b a<_{d} b^{\prime} a^{\prime}$, and if $a b \geqslant_{d} a^{\prime} b^{\prime}$ and $b c>_{d} b^{\prime} c^{\prime}$, then $a c>_{d} a^{\prime} c^{\prime}$, for any $a, b, c, a^{\prime}, b^{\prime}, c^{\prime} \in A$. Let $w, x, y, z \in A$ satisfy $w x \geqslant_{d} x z, w y \leqslant_{r} y z$ and $x y>_{r} x x$. By (i), $x y>_{d} x x$. Now $w x \geqslant_{d} w x$ and $x y>_{d} x x$ imply $w y>_{d} w x$, and $z x \geqslant_{d} z x$ and $x y>_{d} x x$ imply $z y>_{d} z x$, i.e., $x z>_{d} y z$. Hence $w y>_{d} w x \geqslant_{d} x z>_{d} y z$. But then $w y>_{d} y z$ and $w y \leqslant r y z$, so $\geqslant_{d}$ and $\geqslant_{r}$ are not identical.

Q.E.D.

The ratio/difference representation can now be axiomatized using conditions (i) and (ii) of Definition 2 and the condition formulated in Lemma 2.

THEOREM 2. Let $\geqslant_{r}$ and $\geqslant_{d}$ be quaternary relations on an arbitrary set $A$ such that $\left(A \times A, \geqslant_{r}\right)$ and $\left(A \times A, \geqslant_{d}\right)$ are both algebraic difference structures. Then there exists a ratio/difference representation $f$ for $\geqslant_{r}$ and $\geqslant_{d}$ iff the following conditions (i)-(iii) hold: for any $x, y, z, x^{\prime}, y^{\prime}, z^{\prime} \in A$,

\footnotetext{
"See footnote 2 for the definition of "dense in an interval of real numbers."
} 
(i) $x y \geqslant_{r} x x$ iff $x y \geqslant_{d} x x$,

(ii) if $x x^{\prime} \sim_{r} y y^{\prime} \sim_{r} z z^{\prime}$, then $x y \geqslant_{d} y z$ iff $x^{\prime} y^{\prime} \geqslant_{d} y^{\prime} z^{\prime}$,

(iii) there exist $w, x, y, z \in A$ such that $w x \geqslant_{d} x z, w y \leqslant_{r} y z, w z \nsim_{d} z z$ and $x y>_{r} x x$.

Proof. First, suppose that $f$ is a ratio/difference representation for $\geqslant_{r}$ and $\geqslant_{d} d$. It is proven in Fnd (p. 153) that the existence of $f$ implies conditions (i) and (ii) of Theorem 2. To prove condition (iii), first note that we are assuming throughout this essay that $\geqslant_{d}$ is nondegenerate in the sense that $a b>_{d} a^{\prime} b^{\prime}$ for some $a, b, a^{\prime}, b^{\prime} \in A$. If $a b \sim_{d} b b \sim_{d} b^{\prime} b^{\prime} \sim_{d} a^{\prime} b^{\prime}$, then $a b \sim_{d} a^{\prime} b^{\prime}$, contradicting $a b>_{d} a^{\prime} b^{\prime}$. Since $b b \sim_{d} b^{\prime} b^{\prime}$, either $a b \chi_{d} b b$ or $a^{\prime} b^{\prime} \mathcal{\alpha}_{d} b^{\prime} b^{\prime}$. In either case, there exist $w, z \in A$ such that $w z \chi_{d} z z$. Choose any such $w$ and $z$, and let $s, t \in \operatorname{Re}^{+}$satisfy $s=[f(w)+f(z)] / 2$ and $t=[f(w) f(z)]^{1 / 2}$. Then $4 s^{2}=f(w)^{2}+2 f(w) f(z)+f(z)^{2}$ and $t^{2}=f(w) f(z)$, so $4 s^{2}-4 t^{2}=[f(w)-f(z)]^{2}>0$. But then $s-t=\left(s^{2}-t^{2}\right) /$ $(s+t)>0$, so $s>t$. Let $\varepsilon=s-t$.

We know from the proof of Theorem 1 that $f(A)$ is a dense subset of an interval of positive real numbers (see Fnd, p. 159). Therefore there exist $x, y \in A$ such that $\varepsilon / 2>s-f(x) \geqslant 0$ and $\varepsilon / 2>f(y)-t \geqslant 0$. Hence $\varepsilon>s-f(x)+f(y)-t$. Since $s-t=\varepsilon, 0>f(y)-f(x)$, i.e., $f(x) / f(y)>1$. Hence $x y>_{r} x x$. From the choice of $s$ and $x, 2 f(x) \leqslant f(w)+f(z)$. Thus, $f(w)-f(x) \geqslant f(x)-f(z)$, i.e., $w x \geqslant_{d} x z$. From the choice of $t$ and $y, f(y)^{2} \geqslant f(w) f(z)$, so $f(w) / f(y) \leqslant f(y) / f(z)$, i.e., $w y \leqslant_{r} y z$. Hence we have proven the existence of $w, x, y, z \in A$ satisfying $w x \geqslant_{d} x z, w y \leqslant_{r} y z, w z \downarrow_{d} z z$ and $x y>_{r} x x$.

Conversely, suppose conditions (i)-(iii) of Theorem 2 hold. By Lemma 2, condition (iii) of Definition 2 follows from condition (iii) of Theorem 2. Since condition (i) and (ii) of Definition 2 and Thcorcm 2 are identical, generalized ratio/difference compatibility is satisfied by $\geqslant_{r}$ and $\geqslant_{d}$. By Theorem 1 , either there exists a ratio/difference representation $f$ for $\geqslant_{r}$ and $\geqslant_{d}$, or there exists a ratio/difference representation $g$ for $\geqslant_{r^{*}}$ and $\geqslant_{d^{*}}$, but not both. Choose $w, x, y, z \in A$ satisfying (iii) of Theorem 2. If $g$ exists, then $g(w)-g(x) \leqslant g(x)-g(z)$, and $g(w) / g(y) \geqslant g(y) / g(z)$. Hence $2 g(x) \geqslant g(w)+g(z)$ and $g(w) g(z) \geqslant g(y)^{2}$. Since $g(w) \neq g(z)$, we have

$$
\begin{aligned}
4 g(x)^{2}-4 g(y)^{2} & \geqslant g(w)^{2}+2 g(w) g(z)+g(z)^{2}-4 g(w) g(z) \\
& \geqslant[g(w)-g(z)]^{2}>0 .
\end{aligned}
$$

Therefore $[g(x)+g(y)][g(x)-g(y)]>0$. Since $g(x)+g(y)>0$, we have $g(x)-g(y)>0$, i.e., $g(x) / g(y)>1$. Hence $x y>_{r^{*}} x x$, or equivalently, $x y<_{r} x x$. But this contradicts $x y>_{r} x x$. Therefore $g$ does not exist, so $f$ exists.

Q.E.D.

Since under the hypotheses of Theorem 2, conditions (i)-(iii) of that theorem are necessary and sufficient for the existence of a ratio/difference representation, they may be regarded as defining a property called "ratio/difference compatibility."

DEFInItion 3. Let $\geqslant_{r}$ and $\geqslant_{d}$ be quaternary relations on an arbitrary set $A$. 
Then, we say that $\geqslant_{r}$ and $\geqslant_{d}$ satisfy ratio/difference compatibility iff $\left(A \times A, \geqslant_{r}\right)$ and $\left(A \times A, \geqslant_{d}\right)$ are algebraic difference structures and conditions (i)-(iii) of Theorem 2 are satisfied.

The terminology makes sense because ratio/difference compatibility is a special case of generalized ratio/difference compatibility and Theorem 2 establishes that ratio/difference compatible relations possess a ratio/difference representation.

The following corollary points out that if there exists a pair of nonequivalent elements for which $\geqslant_{r}$ and $\geqslant_{d}$ midpoints exist, then there is a simple test for whether a ratio/difference representation exists for $\geqslant_{r}$ and $\geqslant_{d}$, or for $\geqslant_{r^{*}}$ and $\geqslant_{d^{*}}$.

COROLLARY 1. Let $\geqslant_{r}$ and $\geqslant_{d}$ be quaternary relations on an arbitrary set $A$ such that $\geqslant_{r}$ and $\geqslant_{d}$ satisfy generalized ratio/difference compatibility. Let $\geqslant_{r^{*}}$ and $\geqslant_{d^{*}}$ be the respective inverse relations to $\geqslant_{r}$ and $\geqslant_{d}$. Suppose that $w, x, y, z \in A$ are any elements satisfying $w x \sim_{d} x z, w y \sim_{r} y z$ and $w z \chi_{d} z z$. Then, $x y \chi_{r} x x$ and

(i) $x y>_{r} x x$ iff there exists a ratio/difference representation $f$ for $\geqslant_{r}$ and $\geqslant_{d}$,

(ii) $x y<_{r} x x$ iff there exists a ratio/difference representation $g$ for $\geqslant_{r^{*}}$ and $\geqslant_{d^{*}}$.

Proof. Let $w, x, y, z \in A$ satisfy $w x \sim_{d} x z, x y \sim_{r} y z$ and $w z \downarrow_{d} z z$. Note that $w x \sim_{d^{*}} x z, w y \sim_{r^{*}} y z$ and $w z \chi_{d^{*}} z z$, by definition of $\geqslant_{r^{*}}$ and $\geqslant_{d^{*}}$. By Theorem 1 , either there exists a ratio/difference representation for $\geqslant_{r}$ and $\geqslant_{d}$, or there exists a ratio/difference representation for $\geqslant_{r^{*}}$ and $\geqslant_{\alpha^{*}}$, but not both. If $f$ exists, then $f(w)-f(x)=f(x)-f(z), \quad f(w) / f(y)=f(y) / f(z)$ and $f(w)-f(z) \neq 0$. Hence $2 f(x)=f(w)+f(z)$ and $f(y)^{2}=f(w) f(z)$, so

$$
4 f(x)^{2}-4 f(y)^{2}=[f(w)-f(z)]^{2}>0 .
$$

Since $f(x)+f(y)>0$, the left side of (8) can be factored to yield $f(x)-f(y)>0$. Thus $f(x) / f(y)>0$, so $x y>_{r} x x$. Conversely, if $x y>_{r} x x$, then $w, x, y, z$ satisfy (iii) of Theorem 2 . Since $\geqslant_{r}$ and $\geqslant_{d}$ satisfy generalized ratio/difference compatibility, the remaining assumptions of Theorem 2 are satisfied. Therefore there exists a ratio/difference representation $f$ for $\geqslant_{r}$ and $\geqslant_{d}$ by Theorem 2 . A completely analogous argument shows that $g$ exists iff $x y>_{r} x x$, i.e., iff $x y<_{r} x x$. Since $f$ or $g$ exists, we have $x y \chi_{r} x x$ in either case.

Q.E.D.

According to Corollary 1 , if $\geqslant_{r}$ and $\geqslant_{d}$ satisfy generalized ratio/difference compatibility and if it is possible to find $\mathrm{a} \geqslant_{d}$ midpoint $x$ and $\mathrm{a} \geqslant_{r}$ midpoint $y$ for some pair of nonequivalent stimuli, then it is easy to establish whether $\geqslant_{r}$ and $\geqslant_{d}$, or $\geqslant_{r^{*}}$ and $\geqslant_{d^{*}}$ possess a ratio/difference representation. If $x$ is strictiy greater than $y$, a ratio/difference difference representation exists for $\geqslant_{r}$ and $\geqslant_{d}$. If $x$ is strictly less than $y$, a ratio/difference representation exists for $\geqslant_{r^{*}}$ and $\geqslant_{d^{*}}$. Furthermore, it must be the case that either $x$ is strictly greater than $y$ or $x$ is strictly less than $y$. Earlier, the heuristic remark was made that the axiomatization of the ratio/difference representation in Fnd falls short of being sufficient because it omits the requirement that the 
$\geqslant_{d}$ midpoints of nonequivalent elements must be strictly greater than the corresponding $\geqslant_{r}$ midpoints. This remark is valid in any case where it can be established that there exists at least one pair of nonequivalent elements for which $a \geqslant_{r}$ midpoint and $\mathrm{a} \geqslant_{d}$ midpoint both exist. If no such pair exists, the weaker condition (iii) of Theorem 2 will do in its stead, although its verbal formulation is not as simple to state.

\section{APPENDIX}

The proof of Lemma 1 given here parallels the logic of the proof given in Fnd (pp. 160-163), with the exception that a correction is substituted at the point where that proof goes wrong. The present proof also differs from that of Fnd in that certain constructions are explicitly formalized here, whereas they are only informally sketched in Fnd. To facilitate comparisons between the present proof and that of Fnd, equations will be numbered in the manner $(k / n)$ to indicate that the equation is the $k$ th equation of the present essay and the $n$th equation of the relevant sections of Fnd (pp. 152-154; 158-163). For example, Eq. (1) will henceforth be referred to as Eq. (1/5) since it is the first equation of this article but Eq. (5) in the indicated sections of Fnd. Equations only appearing in the present article will be numbered in the usual manner, consecutively with the other equations.

Proof of Lemma 1. It is routine to check that functions of the forms (i) and (ii) are continuous, strictly increasing, and satisfy $(1 / 5)$. We must show that these are the only functions that satisfy $(1 / 5)$.

For $t \in \operatorname{Re}^{+}$, define $I_{2}(t)$ and $I_{1}(t)$ by

$$
\begin{aligned}
& I_{2}(t)=\left\{x \in I_{2}: t x \in I_{2}\right\}, \\
& I_{1}(t)=\left\{H(x) \in I_{1}: x \in I_{2}(t)\right\} .
\end{aligned}
$$

If there exist a greatest element $x^{*}$ and a least element $x_{*}$ in $I_{2}$, let $Z=\left\{x^{*} / x_{*}, x_{*} / x^{*}\right\}$. If $x^{*}$ or $x_{*}$ does not exist, let $Z=\varnothing$, where $\varnothing$ denotes the empty set. Define $T=\left\{s \in \operatorname{Re}^{+}: I_{2}(s) \neq \varnothing\right\}-Z$. It is routine to show that $T$ is an interval of real numbers, $s \in T$ iff $s^{-1} \in T$, and for any $s \in T, I_{2}(s)$ and $I_{1}(s)$ are intervals of real numbers. (The reason for the fussy definition of $T$ is that if $s=x^{*} / x_{*}$, then $I_{2}(s)=\left\{x_{*}\right\}$ is not an interval. So $x^{*} / x_{*}$ and $x_{*} / x^{*}$ must be excluded from $T$ if we are to have that $I_{2}(s)$ is an interval for all $s \in T$.)

By a neighborhood of unity, we simply mean an interval containing 1 . The proof depends on constructing a neighborhood of unity having certain desirable properties (namely, equations (9), (11/11), and (12/12)). This neighborhood of unity (denoted $U$ ) can be constructed as follows. Since $I_{2}$ is a positive interval, we can choose $a, b, c, d \in I_{2}$ such that $a>b>c>d$ and $a / b=c / d$. Let $\mu=\sqrt{a b}$ and $\pi=\sqrt{c d}$. Since $a>\mu>\pi>d$, we must have $\mu, \pi \in I_{2}$. Choose $\xi \in \operatorname{Re}^{+}$such that $\xi^{2}=a / b=c / d$. 
Then $\xi_{\mu}=a, \xi^{-1} \mu=b, \xi \pi=c$ and $\xi^{-1} \pi=d$. Hence $\mu, \pi \in I_{2}(\xi) \cap I_{2}\left(\xi^{-1}\right)$. Moreover, if $\xi^{-1}<\alpha<\xi$ and $x \in I_{2}(\xi) \cap I_{2}\left(\xi^{-1}\right)$, then $\xi^{-1} x<\alpha x<\xi x$ so $x \in I_{2}(\alpha)$. Therefore $I_{2}(\xi) \cap I_{2}\left(\xi^{-1}\right) \subseteq I_{2}(\alpha)$ for every $\alpha$ such that $\xi^{-1}<\alpha<\xi$. Define $U=\{t \in T$ : $\left.\xi^{-1}<t^{2}<\xi\right\}$. Note that if $s, t \in U$, then $\xi^{-1}<s t<\xi$. Hence, st $\in T$ and $I_{2}(\xi) \cap$ $I_{2}\left(\xi^{-1}\right) \subseteq I_{2}(s t)$. Also, if $t \in U$, then $\xi^{-1}<\xi^{-1 / 2}<t<\xi^{1 / 2}<\xi$ so $I_{2}(\xi) \cap I_{2}\left(\xi^{-1}\right) \subseteq$ $I_{2}(t)$. We have established that there exist $\mu, \pi \in I_{2}$ such that $\mu \neq \pi$ and

$$
\mu, \pi \in I_{2}(\xi) \cap I_{2}\left(\xi^{-1}\right) \subseteq I_{2}(s) \cap I_{2}(t) \cap I_{2}(s t)
$$

for every $s, t \in U$.

It can be shown that $(1 / 5)$ implies that

$$
H(t x)=\gamma_{1}(t) H(x)+\gamma_{2}(t)
$$

for all $t \in T$ and $x \in I_{2}(t)$, where $\gamma_{2}$ is some real valued function and $\gamma_{1}$ is a positively valued function (Fnd, p. 161). Furthermore, using (9), it can be shown that $\gamma_{1}$ and $\gamma_{2}$ satisfy the relations

$$
\begin{aligned}
\gamma_{1}(s t) & =\gamma_{1}(s) \gamma_{1}(t), \\
\gamma_{2}(t)\left[\gamma_{1}(s)-1\right] & =\gamma_{2}(s)\left[\gamma_{1}(t)-1\right]
\end{aligned}
$$

for every $s, t \in U$ [Fnd, p. 162]. For the sake of completeness, the derivation of $(10 / 10)-(12 / 12)$ given in Fnd will be repeated here.

For any $t \in T$, define $f_{t}: I_{1}(t) \rightarrow I_{1}$ by $f_{t}(u)=H\left[t \cdot H^{-1}(u)\right]$ for every $u \in I_{1}(t)$. Since $f_{t}$ is a composition of continuous functions, it is continuous. For any $u, v \in I_{1}(t)$, let $x=H^{-1}(u)$ and $z=H^{-1}(v)$; by definition of $I_{1}(t), x, z, t x, t z \in I_{2}$ so by $(1 / 5)$ we have

$$
f_{t}\left[\frac{u+v}{2}\right]=\frac{f_{t}(u)+f_{t}(v)}{2} .
$$

Therefore $f_{t}$ satisfies Jensen's equation; its only continuous solutions on an interval $I_{1}(t)$ have the form $f_{t}(u)=\gamma_{1} u+\gamma_{2}$ (Aczel, 1966, Sect. 2.1.4). Here, $\gamma_{1}$ must be positive since $f_{t}$ is increasing. Noting that $\gamma_{1}$ and $\gamma_{2}$ may depend on $t$ and that $u=H(x)$ for some $x \in I_{2}(t)$, we have $f_{1}[H(x)]=\gamma_{1}(t) H(x)+\gamma_{2}(t)$. Applying the definition of $f_{t}$, we have $(10 / 10)$. Now choose any $s, t \in U$. We know that $t \mu, t \pi \in I_{2}(s)$ and $s \mu, s \pi \in I_{2}(t)$ for the $\mu, \pi$ satisfying (9) because $\mu, \pi \in I_{2}(s) \cap I_{2}(t) \cap$ $I_{2}(s t)$. From $(10 / 10)$ we have

$$
\begin{aligned}
\gamma_{1}(s t)[H(\mu)-H(\pi)] & =H(s t \mu)-H(s t \pi) \\
& =\gamma_{1}(s)\{H(t \mu)-H(t \pi)\} \\
& =\gamma_{1}(s) \gamma_{1}(t)[H(\mu)-H(\pi)] .
\end{aligned}
$$


Since $H$ is strictly increasing, $H(\mu)-H(\pi) \neq 0$. Therefore $(11 / 11)$ holds. Furthermore, repeated application of $(10 / 10)$ yields

$$
\begin{aligned}
H(s t \mu) & =\gamma_{1}(s)\left[\gamma_{1}(t) H(\mu)+\gamma_{2}(t)\right]+\gamma_{2}(s) \\
& =\gamma_{1}(t)\left[\gamma_{1}(s) H(\mu)+\gamma_{2}(s)\right]+\gamma_{2}(t) .
\end{aligned}
$$

Multiplying out this last equation and rearranging terms yields (12/12).

The proof now splits into two cases. First, suppose $y_{1} \equiv 1$ in $U$. Then Eq. $(10 / 10)$ implies that

$$
H(t x)=H(x)+\gamma_{2}(t)
$$

for any $t \in U, x \in I_{2}(t)$. For arbitrarily chosen $s, t \in U$, we may not have $s t \in U$. Nevertheless for the $\mu, \pi \in I_{2}$ satisfying (9), Eqs. (14/13) and (10/10) imply

$$
\begin{aligned}
H(\mu)+\gamma_{2}(t)+\gamma_{2}(s) & =H(t \mu)+\gamma_{2}(s) \\
& =H(s t \mu) \\
& =\gamma_{1}(s t) H(\mu)+\gamma_{2}(s t),
\end{aligned}
$$

and similarly,

$$
H(\pi)+\gamma_{2}(t)+\gamma_{2}(s)=\gamma_{1}(s t) H(\pi)+\gamma_{2}(s t) .
$$

Hence, $H(\mu)-H(\pi)=\gamma_{1}(s t)[H(\mu)-H(\pi)]$. Since $H(\mu) \neq H(\pi), \gamma_{1}(s t)=1$. Combining this with (15) yields

$$
\gamma_{2}(s t)=\gamma_{2}(s)+\gamma_{2}(t)
$$

for any $s, t \in U$.

Second, suppose $\gamma_{1} \neq 1$ in $U$. It is claimed in Fnd (p. 162) that in this case, $\gamma_{1}(t)=1$ iff $\gamma_{2}(t)=0$, but this statement is too strong. If $\gamma_{1}(t)=1$, then $(12 / 12)$ implies that $\gamma_{2}(t)\left[\gamma_{1}(s)-1\right]=0$. Since $s \in U$ may be chosen such that $\gamma_{1}(s) \neq 1$, it follows that $\gamma_{2}(t)=0$. Therefore, $\gamma_{1}(t)=1$ implies that $\gamma_{2}(t)=0$. The converse, however, is not true. Even if $\gamma_{2}(t)=0$, if $\gamma_{2}(s)=0$ for all $s \in U$, then $\gamma_{1}(t)$ need not equal 1. For example, if $H$ is the identity function, then $H$ satisfies $(1 / 5)$, and $H(t x)=\gamma_{1}(t) H(x)+\gamma_{2}(t)$, where $\gamma_{1}$ is the identity function and $\gamma_{2} \equiv 0$. But then for $t \neq 1, \gamma_{2}(t)=0$ but $\gamma_{1}(t) \neq 1$. This error does not lead to invalid inferences in the remainder of the proof presented in Fnd.

If $\gamma_{1} \neq 1$, choose $s, t \in U$ such that $\gamma_{1}(s), \gamma_{1}(t) \neq 1$. Separating variables in $(12 / 12)$ yields

$$
\gamma_{2}(t) /\left[\gamma_{1}(t)-1\right]=\gamma_{2}(s) /\left[\gamma_{1}(s)-1\right]=\beta,
$$


where $\beta \neq 0$ iff $\gamma_{2}(t) \neq 0$ for some $t \in U$ such that $\gamma_{1}(t) \neq 1$. Thus, $\gamma_{2}(t)=\beta\left[\gamma_{1}(t)-1\right]$ for all $t \in U$ (including those $t$ for which $\left.\gamma_{1}(t)=1\right)$. Substituting this in $(10 / 10)$ yields

$$
H(t x)+\beta=\gamma_{1}(t)[H(x)+\beta]
$$

for all $t \in U$ and $x \in I_{2}(t)$.

It is important to examine the sign of $H(x)+\beta$. First suppose that $H(x)+\beta=0$ for some $x \in I_{2}$. Since $I_{2}$ is an interval, there exists $y \in I_{2}$ such that $y<x$ or $x<y$. If $y<x$, choose $t \in U$ such that $y<t x<x$. Then $t x \in I_{2}$, so $(17 / 15)$ implies that $H(t x)+\beta=\gamma_{1}(t)[H(x)+\beta]=0=H(x)+\beta$, contradicting the assumption that $H$ is strictly monotone increasing. Similarly, $x<y$ leads to a contradiction of the same assumption. Hence, $H(x)+\beta \neq 0$ for every $x \in I_{2}$. But now, if $H(x)+\beta<0$ and $H(y)+\beta>0$ for some $x, y \in I_{2}$, then there exists $z \in I_{2}$ such that $H(z)+\beta=0$ because $H$ is continuous and $I_{2}$ is an interval. Since this is impossible, $H(x)+\beta>0$ for all $x \in I_{2}$ or $H(x)+\beta<0$ for all $x \in I_{2}$.

To see that there exists $H$ satisfying (1/5) for which $H(x)+\beta$ is always negative, let $H(x)=-x^{-1}$ for $x \in \mathrm{Re}^{+}$. Then $H$ is a continuous, strictly increasing function satisfying (1/5), $\beta=0$ and $H(x)+\beta<0$ for all $x \in \mathrm{Re}^{+}$. On the other hand, if $H$ is the identity function on $\mathrm{Re}^{+}, H$ satisfies $(1 / 5), \beta=0$ and $H(x)+\beta>0$ for all $x \in \mathrm{Re}^{+}$.

Note that Eqs. $(11 / 11)$ and $(16 / 14)$ are satisfied by all $s, t \in U$. As pointed out in Fnd, these equations are variants of Cauchy's equation whose respective solutions in any neighborhood of unity are

$$
\begin{aligned}
& \gamma_{1}(t)=t^{\theta}, \\
& \gamma_{2}(t)=\eta \log t
\end{aligned}
$$

for some real $\theta$ and $\eta$ (see, also, Aczel, 1966, Sect. 2.1.4). If $\gamma_{1} \equiv 1$ in $U$ and thus $(16 / 14)$ holds, then $\gamma_{2}$ must be strictly increasing because $H$ is strictly increasing and $(14 / 13)$ holds. Thus, $\eta$ in $(19 / 17)$ must be positive.

The critical error in the derivation in Fnd occurs at this point. It is asserted in Fnd that if $\gamma_{1} \not 1$ in $U$, then $\gamma_{1}$ is strictly increasing because $H$ is strictly increasing and $(17 / 15)$ holds. But this inference is valid only if $H(x)+\beta$ is necessarily positive, and as previously noted, this condition is not satisfied. Since $H(x)+\beta$ can either be always positive or always negative, $\gamma_{1}$ can either be strictly increasing or strictly decreasing. Hence, if $\gamma_{1} \equiv 1$, the parameter $\theta$ of $(18 / 16)$ can either be strictly positive or strictly negative depending on the sign of $H(x)+\beta$ (for any $x \in I_{2}$ ).

The remainder of the proof is essentially the same as the proof given in Fnd. Suppose $\gamma_{1} \not \equiv 1$ in $U$. Then from $(18 / 16)$ and $(17 / 15)$ we have

$$
H(t x)+\beta=t^{\theta}[H(x)+\beta]
$$

for all $t \in U$ and $x \in I_{2}(t)$. Choose an arbitrary $x_{0}$ in the interior of $I_{2}$. For any $x \in I_{2}$ such that $x / x_{0} \in U,(20 / 19)$ yields

$$
H(x)+\beta=H\left[\left(x / x_{0}\right) x_{0}\right]+\beta=\left(x / x_{0}\right)^{\theta}\left[H\left(x_{0}\right)+\beta\right]=\alpha x^{\theta}
$$


where $\alpha=x_{0}^{-\theta}\left[H\left(x_{0}\right)+\beta\right]$. To show that (21) actually holds for all $x \in I_{2}$, let $V=\left\{x \in I_{2}: x>x_{0}\right.$ and $\left.H(x)+\beta \neq \alpha x^{\theta}\right\}$. If $V$ is nonempty, let $v$ be the greatest lower bound of $V$. Choose $y \in V$ and $x \in I_{2}-V$ such that $y>v \geqslant x \geqslant x_{0}$ and $y / x \in U$. Since $H(x)+\beta=\alpha x^{\theta},(20 / 19)$ implies that

$$
H(y)+\beta=(y / x)^{\theta}[H(x)+\beta]=\alpha y^{\theta}
$$

contradicting the choice of $y$. Hence $V=\varnothing$. Similarly, let $W=\left\{x \in I_{2}: x<x_{0}\right.$ and $\left.H(x)+\beta \neq \alpha x^{\theta}\right\}$. If $W$ is nonempty, let $w$ be the least upper bound of $W$. Choose $y \in W$ and $x \in I_{2}-W$ such that $y<w \leqslant x \leqslant x_{0}$ and $y / x \in U$. Repeating the previous argument shows that $H(y)+\beta=\alpha y^{\theta}$, contradicting the choice of $y$. Hence $W=\varnothing$. Therefore (21) holds for all $x \in I_{2}$. But this shows that $H(x)=\alpha x^{\theta}-\beta$ for all $x \in I_{2}$. Since $H$ is increasing, the definition of $\alpha$ and (21) imply that $\alpha>0$ iff $H\left(x_{0}\right)+\beta>0$ iff $\theta>0$. Let $\delta=+1$ or -1 depending on whether $H\left(x_{0}\right)+\beta>0$ or $<0$. Then we can stipulate that $\alpha$ and $\theta$ be positive, and $H(x)=\delta \alpha x^{\delta \theta}-\beta$.

If $\gamma_{1} \equiv 1$ in $U$, then from $(14 / 23)$ and $(19 / 17)$ we have

$$
H(t x)=H(x)+\eta \log t
$$

for $t \in U, x \in I_{2}(t)$. Again choosing an arbitrary $x_{0}$ in the interior of $I_{2}$ and any $x \in I_{2}$ such that $x / x_{0} \in U$,

$$
\begin{aligned}
H(x) & =H\left[\left(x / x_{0}\right) x_{0}\right]=H\left(x_{0}\right)+\eta\left[\log x / x_{0}\right] \\
& =\eta \log x+\alpha
\end{aligned}
$$

where $\alpha=H\left(x_{0}\right)-\eta \log x_{0}$. But the same argument used to show that (21) holds for all $x \in I_{2}$ when $\gamma_{1} \neq 1$ in $U$ proves that (22) holds for all $x \in I_{2}$ when $\gamma_{1} \equiv 1$ in $U$.

Q.E.D.

\section{REFERENCES}

ACZEL, J. (1966). Lectures on functional equations and their applications. New York: Academic Press. BIRNBAUM, M. H. (1978). Differences and ratios in psychological measurement. In N. J. Castellan, Jr. \& F. Restle (Eds.). Cognitive theory. (Vol. 3). Hillsdale, N. J.: Erlbaum.

Birnbaum, M. H. (1980). Comparison of two theories of "ratio" and "difference" judgments. Journal of Experimental Psychology: General, 109, 304-319.

Birnbaum. M. H. (1982). Controversies in psychological measurement. In B. Wegener (Ed.), Social attitudes and psychophysical measurement (pp. 401-485). Hillsdale, N. J.: Erlbaum.

Birnbaum, M. H., \& Elmasian, R. (1978). Loudness "ratios" and "differences" involve the same psychophysical operation. Perception \& Psychophysics, 23, 403-408.

Birnbaum, M. H., \& Mellers, B. A. (1978). Measurement and the mental map. Perception \& Psychophysics, 23 (5), 403-408.

Birnbaum, M. H., \& VeIT, C.T. (1974). Scale convergence as a criterion for rescaling: Information integration with difference, ratio and averaging tasks. Perception \& Psychophysics, 15, 7-15.

FAGOT, R. F. (1963). On the psychophysical law and estimation procedures in psychophysical scaling. Psychometrika, 28, 145-160. 
Garner, W. R. (1954). A technique and a scale for loudness measurement. Journal of the Acoustical Society of America, 26 (1), 73-88.

Hagerty, M., \& Birnbaum, M. H. (1978). Nonmetric tests of ratio vs subtractive theories of stimulus comparison. Perception \& Psychophysics, 24, 121-129.

Krantz, D. H., LuCE, R. D., Suppes, P., \& TVersky, A. (1971). Foundations of measurement (Vol. 1) New York: Academic Press.

Marks, L. E. (1974). On scales of sensation: Prolegomena to any future psychophysics that will be able to come forth as science. Perception \& Psychophysics, 16, 358-376.

Mellers, B. A., Davis, D. M., \& Birnbaum, M. H. (1984). Weight of evidence supports one operation for "ratios" and "differences" of heaviness. Journal of Experimental Psychology: Human Perception \& Performance, 10, 216-230.

Parker, S., Schneider, B., \& Kanow, G. ((1975). Ratio scale measurement of the perceived length of lines. Journal of Experimental Psychology: Human Perception and Performance, 104, 195-204.

RULE, S. J., \& CuRTIS, D. W. (1980). Ordinal properties of subjective ratios and differences: Comment on Veit. Journal of Experimental Psychology: General, 109, 296-300.

Rule, S. J., Curtis, D. W. \& Mullin, L. C. (1981). Subjective ratios and differences in perceived heaviness. Journal of Experimental Psychology: Human Perception and Performance, 7, 459-466.

Schneidek, B., Parker, S., Farrell, G., \& Kanow, G. (1976). The perceptual basis of loudness ratio judgments. Perception \& Psychophysics, 19, 309-320.

Stevens, S. S. (1957). On the psychophysical law. Psychological Review, 64, 153-181.

Stevens. S. S. (1971). Issues in psychophysical measurement. Psychological Review. 78, 426-450.

TORgERson, W. S. (1961). Distances and ratios in psychophysical scaling. Acta Psychologica, 19 (5). 201-205.

VEIT, C. T. (1978). Ratio and subtractive processes in psychophysical judgment. Journal of Experimental Psychology: General, 107, 81-107.

VEIT, C. T. (1980). Analyzing "ratio" and "difference" judgments: A reply to Rule and Curtis. Journal of Experimental Psychology: General, 109, 301-303.

RECEIVED: September 21, 1983 